\title{
Length of DNA Linker Affects Nucleosomal DNA Structure
}

Tatiana Andreeva ${ }^{1}$, Natalya Maluchenko ${ }^{1}$, Oleg Chertkov ${ }^{1}$, Vasily Studitsky ${ }^{2}$, Alexey Feofanov ${ }^{3}$ and Mikhail Kirpichnikov ${ }^{3}$

${ }^{1}$ Lomonosov Moscow State University, Moscow, Moskva, Russia, ${ }^{2}$ Fox Chase Cancer Center, Lomonosov Moscow State University, Philadelphia, Pennsylvania, United States, ${ }^{3}$ Lomonosov Moscow State University, Shemyakin-Ovchinnikov Institute of Bioorganic Chemistry, Moscow, Moskva, Russia

A nucleosome is a structural unit of a chromatin organization formed by a DNA fragment of 147 bp length wrapped around a histone octamer (two H2A-H2B dimers and (H3-H4)2 tetramer). Structural changes in nucleosomes modulate interactions of nuclear proteins with nucleosomal DNA and efficiency of functionally important processes such as DNA reparation, transcription and replication. Factors affecting the structural organization of nucleosomal DNA (and thereby DNA processing) require detailed investigation. One of the new informative methods for studying a nucleosome structure is the single particle microscopy based on the Förster resonance energy transfer (spFRET microscopy) [1]. This method allows one to get information about the structure of thousands of nucleosomes characterizing each of them individually (Fig. $1 \mathrm{~A})$.

We investigated an effect of a length of a DNA linker on the structure of nucleosomal DNA in the absence and presence of magnesium ions. Two types of nucleosomes were studied: nucleosomes with a short linker of 20 bp length (LN20) and nucleosomes with a long linker of 135 bp length (LN135, Fig. 1). Nucleosomal DNA was labelled by Cy3 and Cy5 fluorophores at positions +13 and +91 bp (numbering starts from the beginning of nucleosome positioning sequence). LN20 are commonly used in our spFRET studies of nucleosome complexes with nuclear proteins [2-4], while LN135 were specially designed to carry out investigations of nucleosome transcription with RNA polymerase [5,6]. Fluorescence of single nucleosomes was measured during their free diffusion through the laser beam focus, proximity ratio EPR (a close analog of FRET efficiency) was calculated for each nucleosome, and accumulated data sets were presented as the frequency distributions of nucleosomes by EPR (Fig. 1 A). The EPR profiles of LN20 and LN135 nucleosomes (Fig. 1 B, D) were fitted by two Gaussians, and fractions of nucleosomes possessing low EPR (LF) and high EPR (HF) were calculated (Fig. 1 C, E). HF fraction contains nucleosomes, where DNA is tightly wrapped over a histone octamer, and the distance between neighbouring DNA gyres is small at the labelled sites. In the LF fraction, DNA is unwrapped from a histone octamer near the nucleosome boundary probably due to so called "nucleosome breathing".

Data of a spFRET microscopy indicate that the structure of nucleosomal DNA differs in LN20 and LN135. It is manifested by a shift of the HF fraction EPR maximum from 0.6 (LN20) to 0.65-0.7 (LN135) as well as by narrowing of the EPR distribution for LN135 as compared to that of LN20 (Fig. 1 B, D). These changes can be interpreted as mutual approaching of DNA gyres at the labelled sites and restriction of DNA gyre dynamics in LN135. Structural changes in LN135 as compared to LN20 can be induced by a stronger interaction of a long DNA linker with histone tails.

Addition of $\mathrm{Mg}^{2+}$ does not eliminate the structural differences between LN20 and LN135 (Fig. 1 B, D). $\mathrm{Mg}^{2+}$ ions do not affect noticeably the LN135 structure near a nucleosome boundary (Fig. 1 D), but do increase a LF fraction of LN20 (Fig. 1 B, C) highly probably by competing with core histones for the 
binding with DNA near the entrance of DNA into nucleosome and enhancing in this way a "nucleosome breathing".

In conclusion, the found structural differences of LN20 and LN135 can affect stability of nucleosomes as well as their interactions with nuclear proteins, and corresponding studies are in progress now [7].
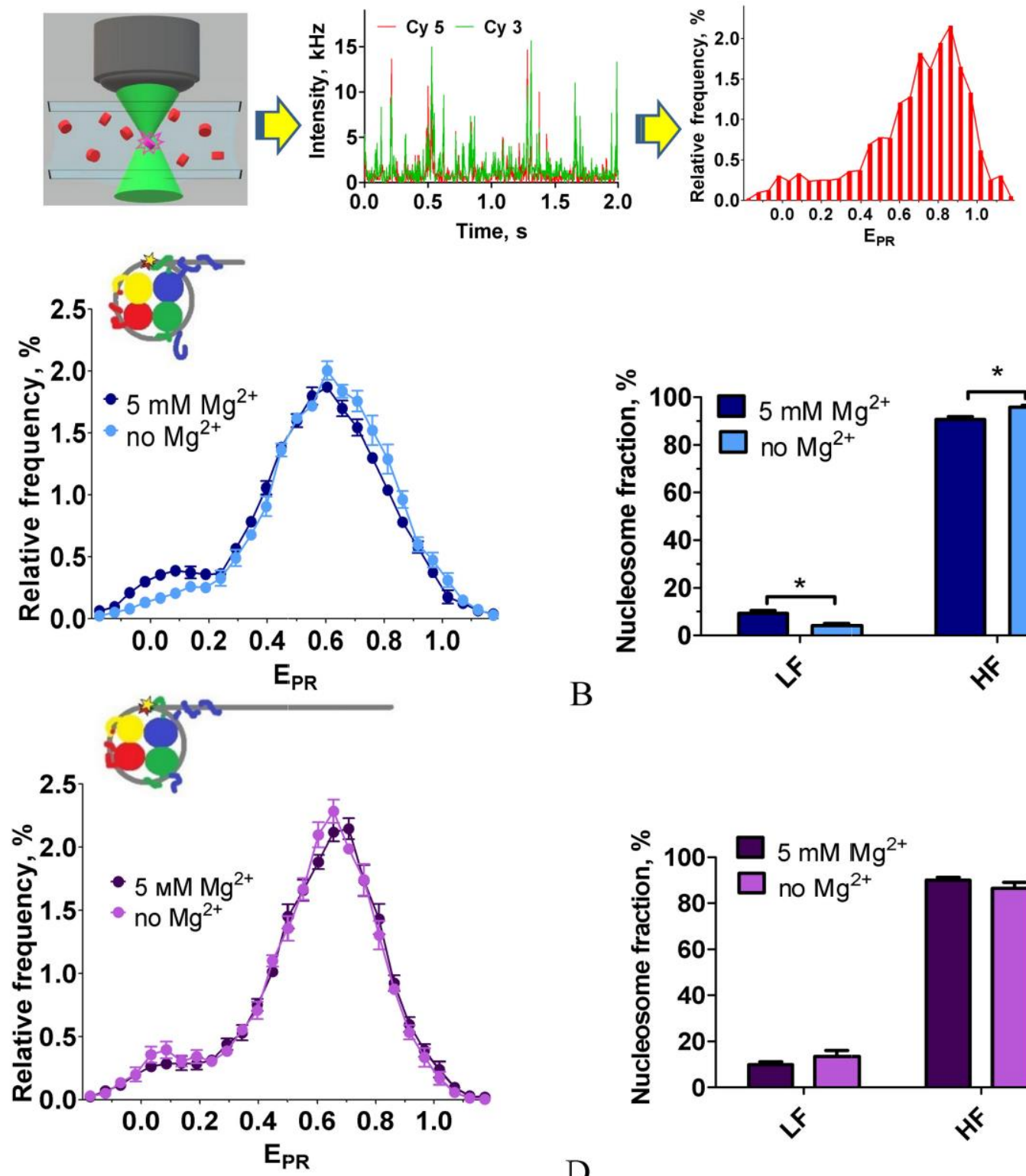

B

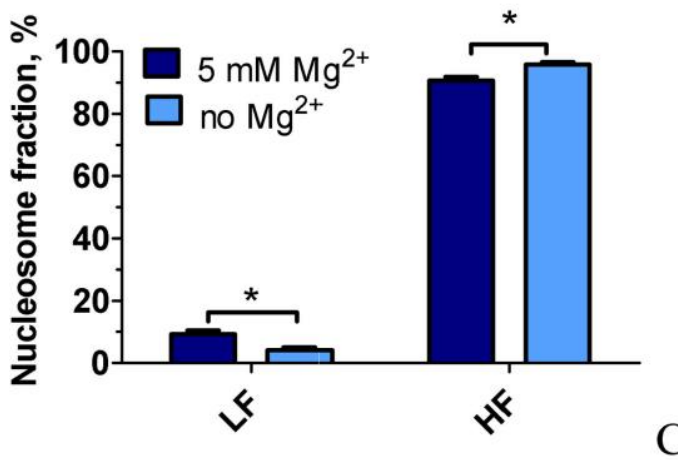

Figure 1. spFRET microscopy study (A) of nucleosomes LN20 (B, C) and LN135 (D, E). (A) Scheme of spFRET experiment and result analysis. (B, D) Relative frequency of nucleosome distribution by EPR value in the presence and absence of $5 \mathrm{mM} \mathrm{MgCl} 2$ (mean $\pm \mathrm{SEM}, \mathrm{n}=3$ ). Buffer $-150 \mathrm{mM} \mathrm{KCl}, 20 \mathrm{mM}$ Tris $\mathrm{HCl}, \mathrm{pH}=8$.0. Inserts - structures of the studied nucleosomes and positions of labels (asterisks). (C, E) Comparison of the $\mathrm{LF}$ and $\mathrm{HF}$ fractions of nucleosomes in the presence and absence of $5 \mathrm{mM} \mathrm{MgCl} 2$. *p $<0.05$ 


\section{References}

[1] R. Buning, et al. Biochimie 92 (2010) P.1729-1740.

[2] M. Valieva et al. Nat. Struct. Mol. Biol. 23 (2016) P. 1111-1116.

[3] H.-W. Chang et al. Science Advances 4 (2018) P. eaav2131.

[4] D. Sultanov et al. AIMS Genetics 4 (2017) P. 21-31.

[5] O.I. Kulaeva et al. Nat. Struct. Mol. Biol. 16 (2009) P. 1272-1278.

[6] K. Kudryashova et al. Methods Mol Biol. 1288 (2015) P. 395-412.

[7] Financial support by Russian Science Foundation (grant 19-74-30003) is acknowledged. 